Research article

\title{
Secretion of full-length tau or tau fragments in a cell culture model
}

\author{
Mar Pérez ${ }^{\mathrm{a}}$, Raquel Cuadros ${ }^{\mathrm{b}, \mathrm{c}}$, Félix Hernández ${ }^{\mathrm{b}, \mathrm{c}}$, Jesús Avila ${ }^{\mathrm{b}, \mathrm{c}, *}$ \\ a Departamento de Anatomía Histología y Neurociencia, Facultad de Medicina UAM, 28029 Madrid, Spain

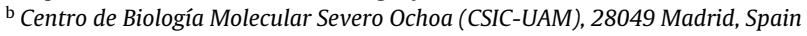 \\ c Centro de Investigación Biomédica en Red de Enfermedades Neurodegenerativas (CIBERNED), 28031 Madrid, Spain
}

\section{H I G H L I G H T S}

- Using different tau construct we have described different mechanisms for tau secretion in transfected COS-7 cells.

- N-terminal region of tau molecule without the microtubule binding repeats is secreted.

- Tau peptide with three microtubule binding repeats is not secreted.

- Tau peptide formed by three microtubule binding repeats plus the C-terminal end is secreted to the extracellular medium.

\section{A R T I C L E I N F O}

\section{Article history:}

Received 21 July 2016

Received in revised form

14 September 2016

Accepted 16 September 2016

Available online 17 September 2016

\section{Keywords:}

Tau protein

Extracellular

Tau fragments

Protein secretion

Protein aggregation

\begin{abstract}
A B S T R A C T
Tau is a microtubule-associated protein that plays an important role in the pathogenesis of neurodegenerative diseases such as Alzheimer's disease. Several studies have suggested that tau may be secreted to extracellular medium and may be responsible of spreading of neurodegeneration. The overexpression of tau in cultured non-neuronal cells leads to the secretion of this protein. The proline-rich region of tau may serve as a membrane-binding site during the secretion of the full-length tau molecule. Tau fragments lacking this proline-region are either not secreted or are secreted in a distinct manner to the full-length molecule.
\end{abstract}

(C) 2016 Elsevier Ireland Ltd. All rights reserved.

\section{Introduction}

Tau protein is a brain microtubule-associated protein found mainly in the cytoplasm of neurons bound to microtubules [43]. Although tau is an intracellular protein, upon neuron death it can also be present in the extracellular space [20], being detected in human cerebrospinal fluid $[4,7,39]$. Later on, it was reported that tau protein is secreted in the absence of cell death $[11,16,21,41]$ but that such secretion is not related to the conventional mechanism used by constitutively secreted proteins $[14,23,29]$. Thus, secretion may depend on a specific factor that promotes the release of tau. One of these factors is the accumulation of intracellular tau, which occurs in some diseases through an increase in the synthesis [5] or a decrease degradation [45] of this protein.

\footnotetext{
* Corresponding author at: Centro de Biología Molecular Severo Ochoa (CSICUAM), 28049 Madrid, Spain.

E-mail addresses: javila@cbm.csic.es, jesus.avila@csic.es (J. Avila).

URL: http://mailto:javila@cbm.csic.es (J. Avila).
}

One approach to mimic intracellular tau accumulation is by overexpressing tau in non-neuronal cells. Under these conditions, tau is secreted when the amount of intracellular tau reaches a certain level $[11,41]$. Extracellular tau can be released from different non-neronal cell lines and neurons via multiple pathways. Thus, it has been shown that tau can be secreted in naked form [11] or associated to exosomes [40] and/or other membrane vesicles [41]. The main difference with non-neuronal cells is that in mature neurons tau can be stimulated by neuronal activity $[15,28,32,33,37,38,40]$. Indeed, under normal circumstances, extracellular tau is found in brain interstitial fluid [44]. However, tau secretory pathways remain to be fully characterized.

Also, it has been proposed that pathological conditions are characterized by abnormal tau secretion, as this phenomenon occurs in tauopathies such as Alzheimer's disease (AD). Thus, abnormal secretion has been reported for modified full-length tau and for some fragments of this protein, the latter mainly in an aggregated form in vesicles $[9,16]$ or in nacked form [40]. The presence of fragments containing $\mathrm{N}$-terminal regions has been reported in CSF $[1,40]$. In addition, it has been described that a $24-\mathrm{kDa}$ fragment 
lacking 242 residues at its $\mathrm{N}$-terminal region is secreted in some tauopathies [10,30]. Moreover, deletion of the C-terminal region at residue 421 may enhance tau aggregation and secretion [36], and C-terminal truncated tau is released into the extracellular medium [42]. Also, C-terminal truncated forms but not full-length tau is released in the absence of cell death in cultured neurons [26]. In addition, several truncated tau fragments lacking the N-terminus and/or C-terminus fragments have been observed in the AD brain [39].

Here we addressed the secretion of full-length tau or tau fragments truncated at their $\mathrm{N}$-terminal or C-terminal regions in a non-neuronal cell model.

\section{Materials and methods}

\subsection{Antibody characterization}

Antibody Tau 12 (N-terminal, mouse monoclonal; Abcam) recognizes phosphorylated and unphosphorylated human Tau. Antibody Tau 46 (C-terminal, mouse monoclonal; Abcam) recognizes phosphorylated and unphosphorylated human Tau. Antibody Anti-Tau 3RD (3-repeat isoform RD3; Millipore) recognizes isoforms of Tau with 3 microtubule binding repeats. Monoclonal antibody PHF-1 recognizes tau phosphorylated at Ser396/404 (Kindly provided by Dr. Peter Davies). Antibody against $\beta$-actin was obtained from Sigma.

\subsection{Materials}

The anti-proteases cocktail was purchased from Sigma-Aldrich. Okadaic acid was obtained from Calbiochem.

\section{3. cDNA tau constructs}

All tau cDNA constructs were cloned into the eukaryotic expression vector pSG5 (Stratagene) under the control of SV40 early promoter. Plasmids pSGT30, pSGT42, pSGT2N, pSGT3RC and pSGT3R have been previously described [34]. Tau isoform nomenclature defines the length of the microtubule binding repeat (3R or $4 \mathrm{R}$ ) and the presence or absence of exons 2 and 3 at the N-terminus ( 0 or 2 ). Plasmid pSGT2N corresponds to the fragment of tau that contains the N-terminus with exons 2 and 3. Plasmids pSGT3RC and pSGT3R contain 3 microtubule binding repeat and or no the C-terminus of tau molecule, respectively.

\subsection{Cell culture, DNA transfection and protein extracts}

African green monkey kidney fibroblasts (COS-7) cells [17] were grown in Dulbecco's modified Eagle's medium supplemented with $10 \%$ (vol/vol) fetal bovine serum (FBS). Cells were transfected with the plasmids described above [31] using PEI reagent (Polysciences, Inc) according to the manufacturer's instructions. Control cells were transfected with the empty vector pSG5 (Stratagene). At $48 \mathrm{~h}$ post-transfection, the cells were harvested in chilled phosphatebuffered saline (PBS), resuspended and homogenized in buffer containing $20 \mathrm{mM}$ HEPES pH 7.4, $5 \mathrm{mM}$ EDTA, $100 \mathrm{mM} \mathrm{NaCl}, 1 \%$ Triton X-100, $0.1 \mathrm{mM}$ sodium orthovanadate, 1x cocktail of antiproteases and 1/1000 Okadaic acid. Lysates were centrifugated at $10,000 \mathrm{~g}$ for $10 \mathrm{~min}$ at $4{ }^{\circ} \mathrm{C}$ and boiled in Laemmli's buffer. Protein samples were quantified by the BCA protein assay.

\subsection{Measuring tau release from $\mathrm{COS}-7$ cells}

The protocol for production of tau conditioned medium is described in [41]. Briefly, the culture media of the cell cultures were collected after $48 \mathrm{~h}$ post-transfection. Conditioned medium was isolated as described by centrifugation at $300 \times \mathrm{g}$ for $10 \mathrm{~min}$. The supernatant was then centrifuged at $2000 \times \mathrm{g}$ for $10 \mathrm{~min}$, and the new supernatant was centrifuged at $10,000 \times g$ for $30 \mathrm{~min}$ at $4{ }^{\circ} \mathrm{C}$ to remove cell debris. This supernatant was called Final supernatant. In some experiments, in order to separate polymerized protein from unpolymerized protein, $100 \mu$ l of final supernatant was centrifuged again in an Airfuga (Beckman) for $30 \mathrm{~min}$ at room temperature at maximum speed, and the pellet and resulting supernatant were collected.

\subsection{Western blotting}

Samples were run on $10 \%$ SDS-PAGE and electrophoretically transferred to a nitrocellulose membrane (Schleicher\&Schuell $\mathrm{Gmb}$ ). The membrane was blocked by incubation with $5 \%$ semifat dried milk in PBS and 0.1\% Tween 20 (PBSM), followed by 1 -h incubation at room temperature with the primary antibody in PBSM. The following primary antibody dilutions were used: T12 (1/500); T46 (1/1000); 3RD (1/1000); PHF-1 (1/100); anti- $\beta$ actin (1/2000). After three washes, the membrane was incubated with a horseradish peroxidase-anti-mouse Ig conjugate (DAKO), followed by several washes in PBS-Tween 20. The membrane was then incubated for $1 \mathrm{~min}$ in Western Lightning reagents (PerkingElmer Life Sciences).

\subsection{Toxicity assays}

Cell death was assayed by using the LIVE/DEAD viability/cytotoxicity kit (Invitrogen, Carlsbad, CA), that contains calcein AM, that only labels live cells, and ethidium homodimer-1, that enters cells with loss of plasma membrane integrity (dead cells). For analyze the cell death, $1 \times 10^{5} \mathrm{COS}-7$ cells were seeded to each well of a 24-well plate and transfected with the plasmids described above. After $48 \mathrm{~h}$ postransfection, cell viability was measured using LIVE/DEAD viability kit. Briefly, cells were incubated for $20 \mathrm{~min}$ with $2 \mu \mathrm{M}$ propidium iodide and $1 \mu \mathrm{M}$ calcein. After staining, live cells (green) and dead cells (red) were visualized on a Leica fluorescence microscope and images were taken. Three fields (selected at random) were analyzed per well (1000-2000 cells/field) and counted with Image J software. Cell death was defined in each condition as the percentage of dead cells versus the total number of cells.

\section{Results}

\subsection{Secretion of tau isoforms in nacked form}

In tauopathies, such as $\mathrm{AD}$, the amount of intracellular tau is increased compared with a non-pathological situation [25]. This increase could be due to a higher synthesis of tau [5] or to a lower degradation of the protein [45]. One approach to promote an increase in intracellular tau in cultured cells is through the use of transfected cells overexpressing the protein [11,41].

Brain tau contains six isoforms; the longest one contains exons 2, 3 and 10 whereas the smallest carries none of these exons [19]. In $\mathrm{AD}$ brains all six isoforms are presented in filaments, but in other tauopathies tau isoforms with four or three repeats are found [18]. Using transfected COS-7 cells, we studied whether the longest (T42) and shortest isoforms (T30) (Fig. 1a) are secreted in a similar form. We analyzed by Western blot the amounts of intracellular and extracellular tau present in the culture medium (Fig. 1) and found that intracellular tau level for both tau isoforms is very similar when secretion of the protein takes place. This result indicates that exons 2, 3 and 10 exert a low influence, if any, over tau secretion. In addition, we examined a tau variant lacking the first ten residues located at the $\mathrm{N}$-terminus. In this case, we also detected a similar efficiency 
a
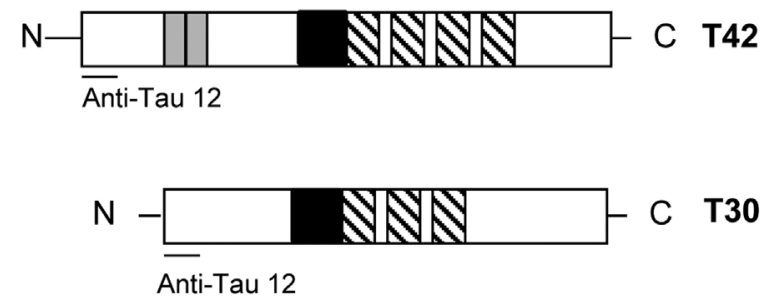

C

\section{Lysate}

Anti- $\beta-$ Actin

Final
supernatant
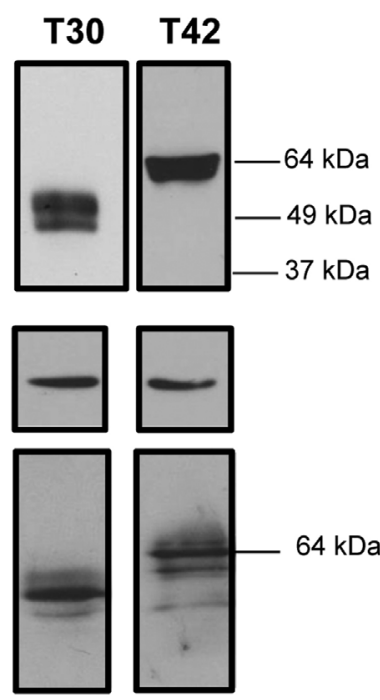

b
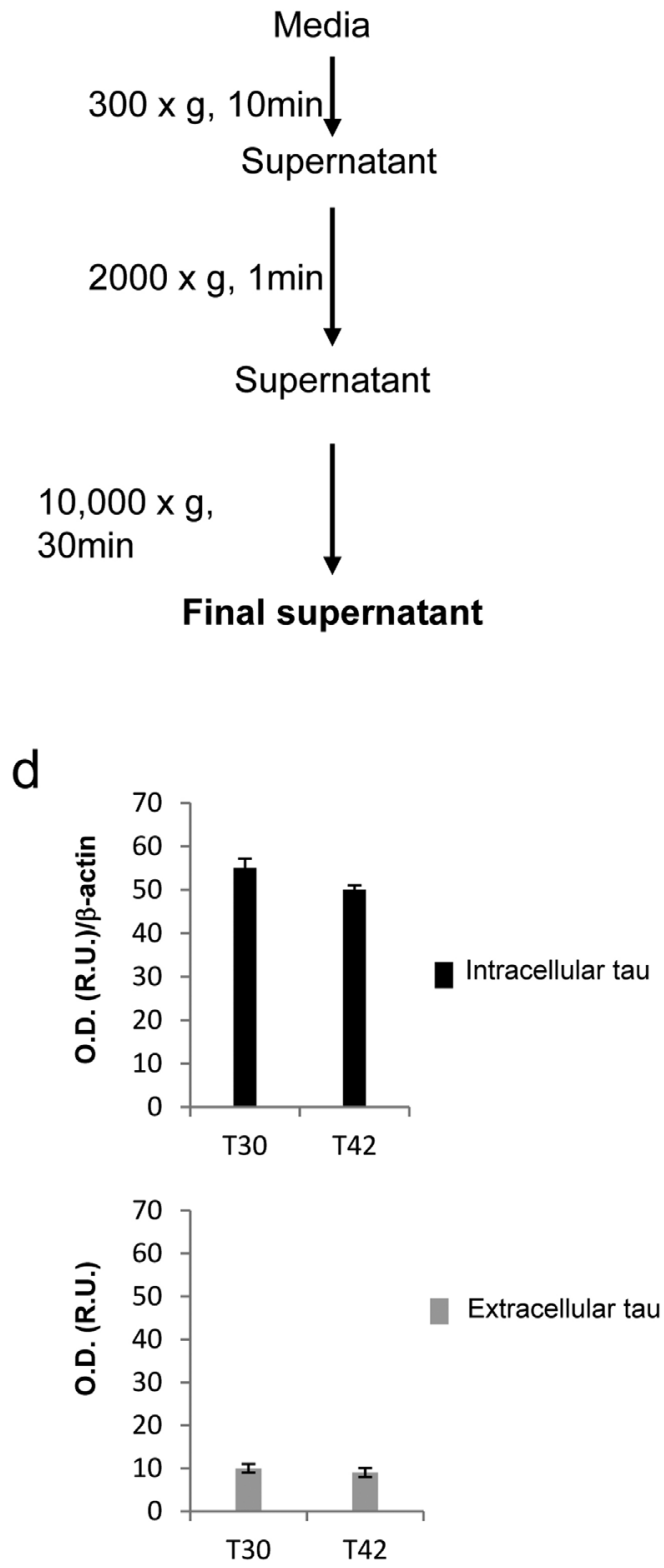

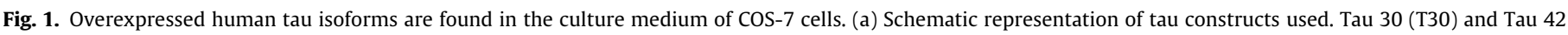

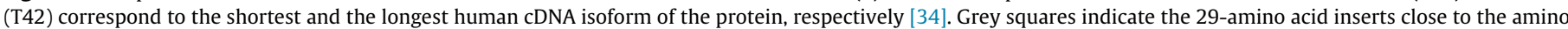

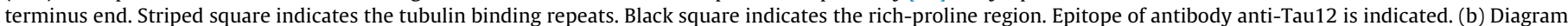

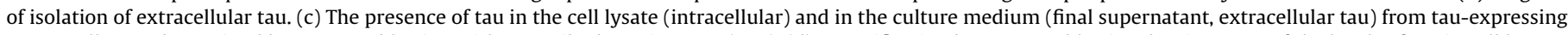

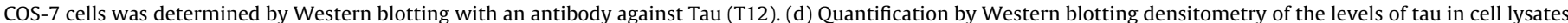

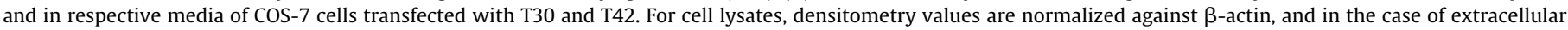
medium, serum albumin, observed by Ponceau's staining, was used as loading protein control. Data are mean \pm SD from six separate experiments ( $\mathrm{n}=6$ ).

of secretion to that of the full-length molecule (see Supplementary Fig. 1 in the online version at DOI: 10.1016/j.neulet.2016.09.026). These observations suggest that the first 103 residues, to exon 3 of tau molecule, do not participate in tau secretion.

\subsection{Secretion of tau fragments}

In order to determine the secretion of tau fragments from neuronal cells, a recent study analyzed the presence of specific fragments in the culture medium using highly sensitive ELISAs with specific antibodies raised against particular regions of the molecule [26]. Most of the secreted fragments were C-terminal truncated, lacking of the microtubule-binding regions (MTBRs), and were enriched in the mid-region, which is rich in proline residues [26]. We therefore tested whether a tau fragment (T3R) bearing MTBRs and lacking the mid-region is secreted with a similar efficiency to that of another tau fragment containing the proline region (T2N). In agreement with previously published data [26], we observed a marked difference between the secretion of T3R (not detected in cultured medium) and T2N (Fig. 2b and c). In order to ensure that we only observed secreted tau, we performed a toxicity assay with tau plasmids used in this work. Table 1 shows that the presence of extracellular tau did not correlated with cell death, given the low rate of cell death observed in the cultures of tau transfected COS-7 cells. 
a
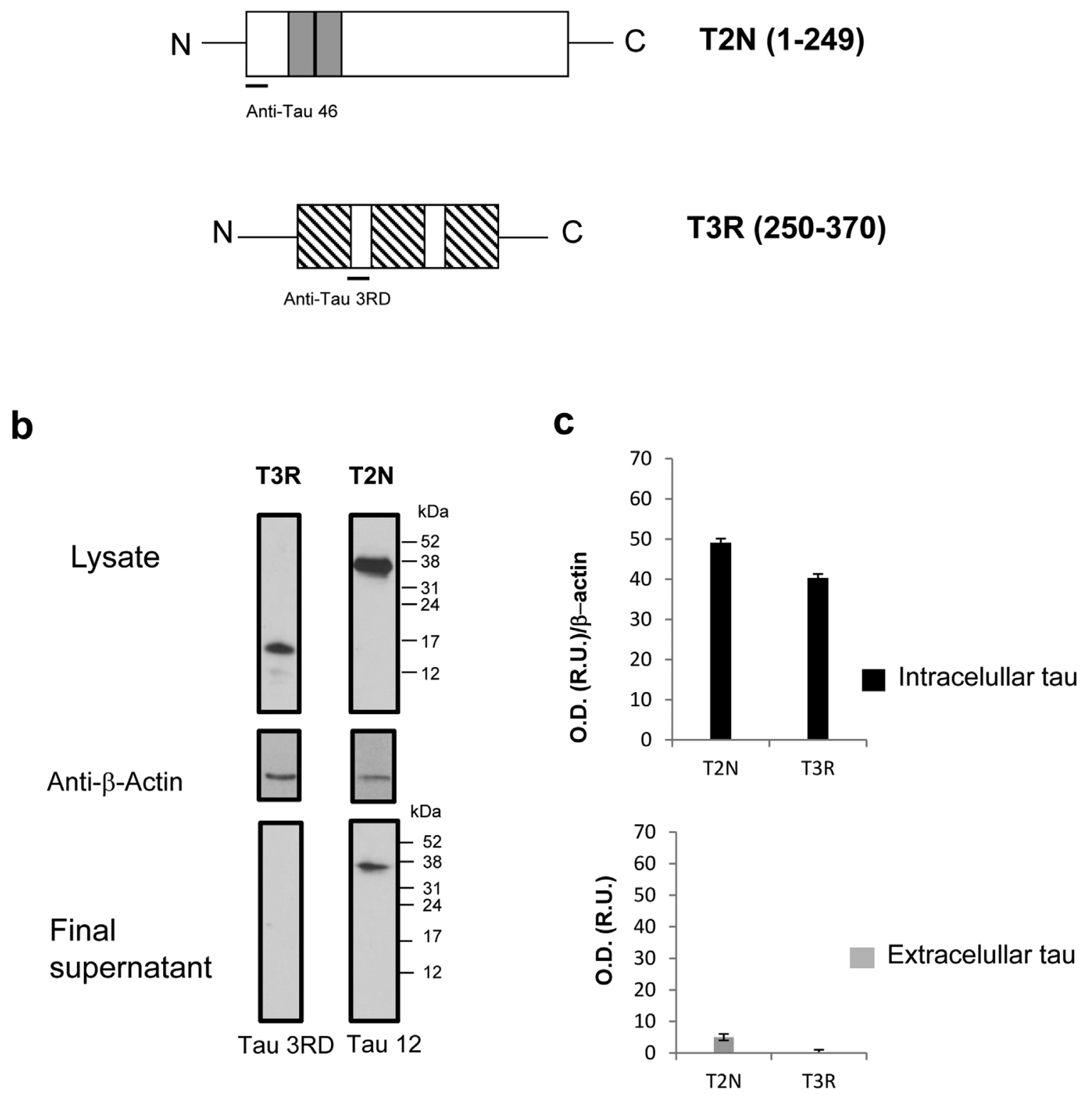

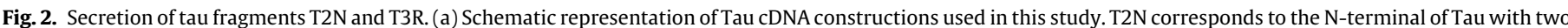

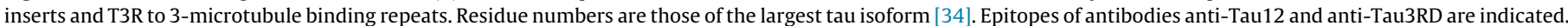

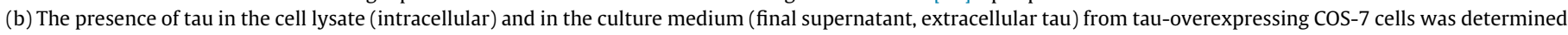

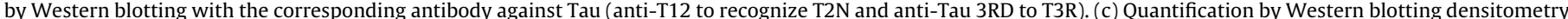

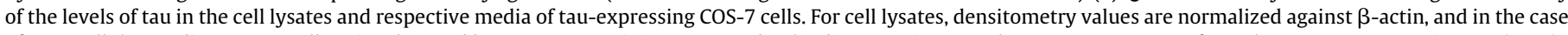

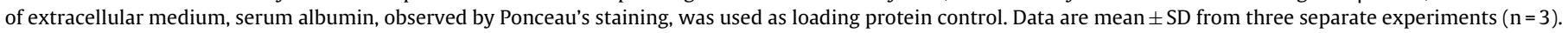

Table 1

Percentage of dead cells.

\begin{tabular}{ll}
\hline Tau Construct & \% Dead Cells \\
\hline pSG5 & $5.8 \pm 2.5$ \\
T42 & $4.8 \pm 1.5$ \\
T30 & $1.7 \pm 2.1$ \\
T2N & $5.0 \pm 1.7$ \\
T3RC & $7.5 \pm 3.5$ \\
T3R & $1.8 \pm 2.5$ \\
\hline
\end{tabular}

\subsection{The aberrant secretion of tau 3RC fragment}

As previously proposed [26], our data also supports the notion that the presence of a proline-rich region facilitates tau secretion
(Fig. 2). To test this hypothesis, we analyzed the secretion of the T3RC fragment (bearing MTBR and C-terminal region) lacking this proline-rich region (see Fig. 3a) as a negative control. Surprisingly, these fragments were also secreted in a similar form to that of T2N fragments (Fig. 3b), thereby suggesting that T3RC fragments are secreted through a mechanism other than proline-rich dependent secretion. However, intracellular T3RC fragments are phosphorylated in PHF-1 domain (Fig. 3b), while extracellular T3RC is not recognize by the antibody PHF-1.

Analysis of the cultured medium of T3RC-transfected cells revealed various polypeptides belonging to T3RC (Fig. 3d). This behavior was not found for the secreted T2N fragment. The secretion of the T3RC fragment may require a previous modification (aggregation or truncation) before it can be released from the cell. 
a

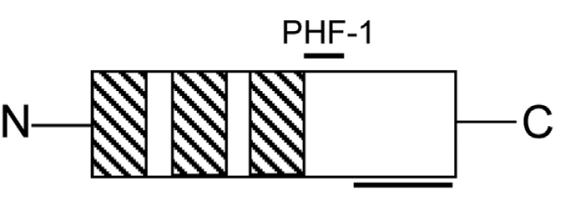

Anti-Tau 46

\section{T3RC (250-441)}

b

\section{C}
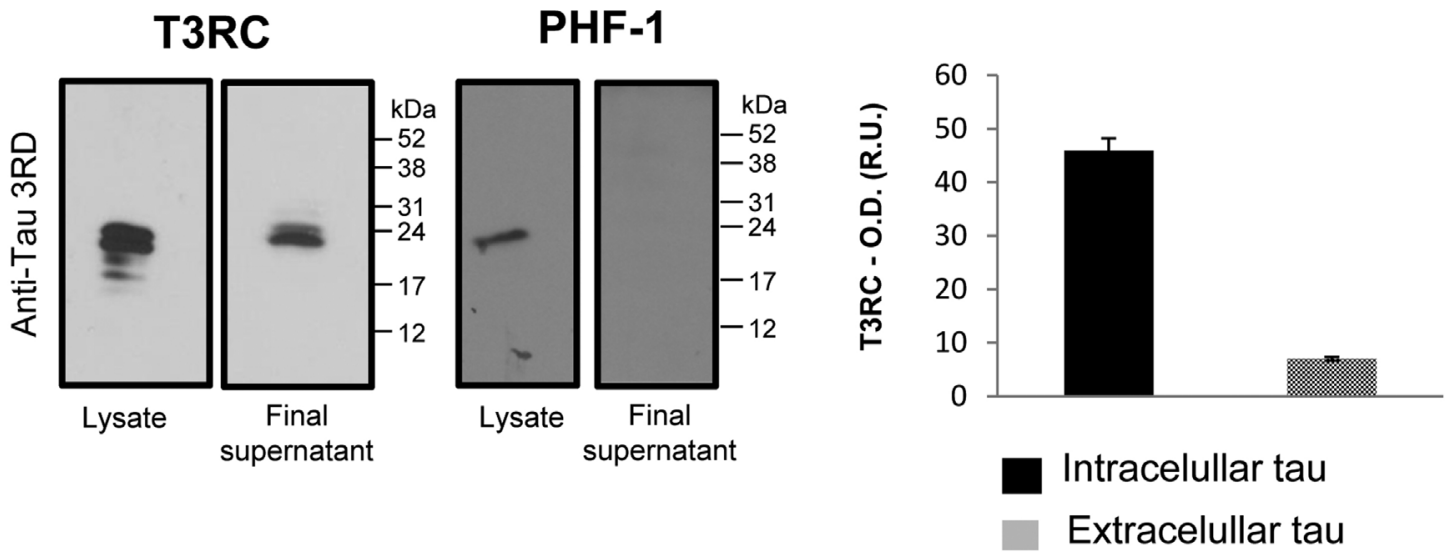

d

T2N

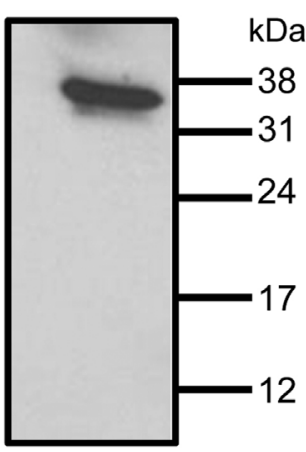

Tau 12

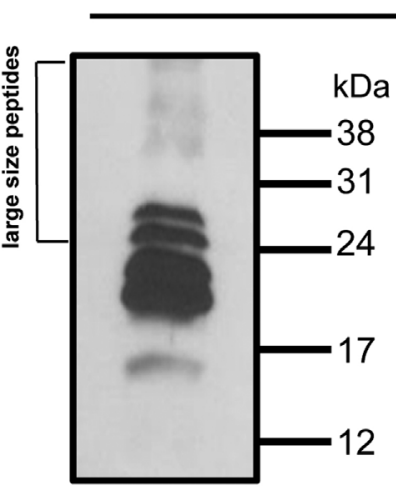

Tau 3RD

T3RC

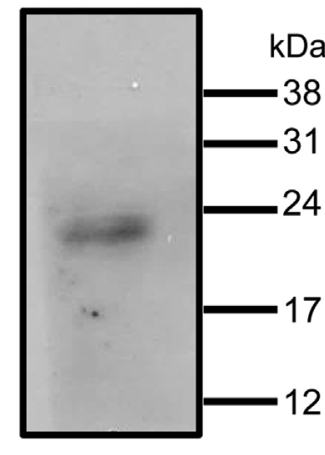

Tau 46

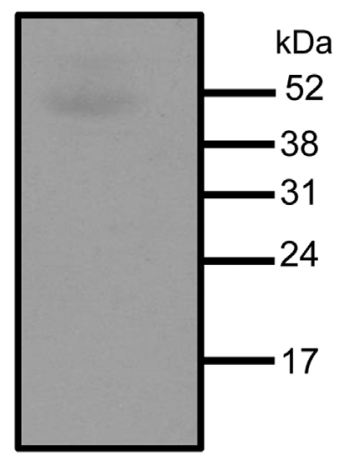

PHF-1

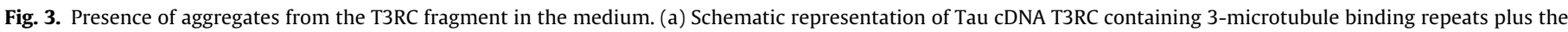

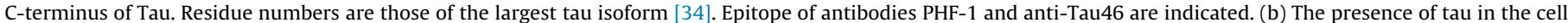

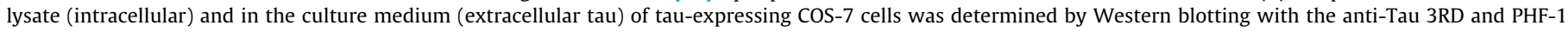

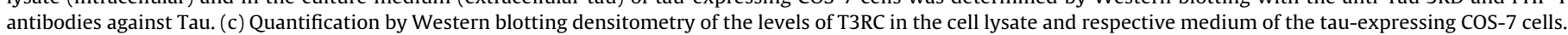

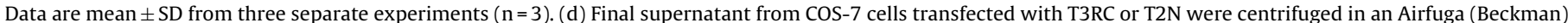

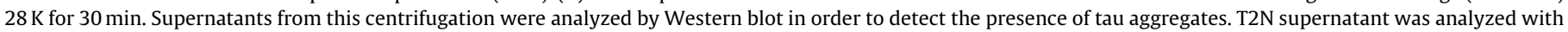
anti-Tau 12 and T3RC supernatants were analyzed with different antibodies: Tau 3RD, Tau 46 and PHF-1. The presence of aggregated T3RC is indicated in the Figure.

Aggregation seems to occur in our cellular model as anti-tau3RD recognizes tau bands with a molecular weight higher than $24 \mathrm{kDa}$ (Fig. 3d). Recent reports have put forward this mechanism for the pathological secretion of tau (or tau fragments) in tauopathies such as $\mathrm{AD}[9,16,26]$. Indeed, deletion (truncation) of the last C-terminal region may take place in our cell model since tau fragments with a molecular weight lower than $24 \mathrm{kDa}$ are observed with 3RD antibody, but did not react with the Tau 46 antibody (Fig. 3d), an antibody reacting with an epitope present in the last 37 residues of the C-terminal of tau [26]. In addition, extracellular T3RC is not phosphorylated as does not react with PHF-1 antibody. Thus, sev- 
eral secretion mechanisms for T2N and T3RC fragments could be proposed.

\section{Discussion}

Although tau is an intracellular protein, it can be found in the extracellular space upon cell death [20]. Alternatively, in the absence of cell death and mainly based on cell models, tau can be released from a cell upon accumulation of intracellular tau [41]. Tau accumulation could be the result of an increased expression or decreased degradation of the protein $[5,45]$. It has recently been reported that impaired degradation is promoted by a modified form of tau itself [45].

Here we used a cell culture system to identify regions of tau involved in the secretion of this protein. Although, it has been previously published that exon 2 modulates Tau secretion [27], taking into account our data, we found that exons 2 and 3 are not involved in tau release as Tau30 and Tau42 showed a similar level of secretion. Theoretically, exon 4 is unlikely to be involved as this exon holds many acidic residues. However, the likelihood that the adjacent proline-rich region is involved is higher since it binds to the cell membrane [8]. In addition, a previous study examining the differences in the secretion of various tau fragments reported a higher proportion of those containing the proline-rich region [26]. That study also described a decrease in the secretion of those fragments containing MTBRs [26]. The latter observation could be attributable to the preferential retention of tau in the cell cytoplasm as a result of its binding to microtubules. In the first case, as indicated, tau could bind to membrane proteins through its proline-rich region $[2,8]$, which contains motifs like PPXXP or PXXP. These motifs, in turn, may interact with proteins containing SH3 domains $[3,24]$. The binding of tau to cell membranes may be the first step required for tau secretion.

In addition to proline-rich region-dependent secretion, the secretion of fragments of the protein follows a distinct pathway, which may involve a previous modification of tau peptides by aggregation or truncation-modifications that may occur in tauopathies such as AD [6,9]. Truncations at the N-terminal or C-terminal regions that result in toxic tau fragments have been reported $[12,35,45]$. Here we show that secretion of T3RC shows several differences with the other mode of secretion. This enhancement may be explained by previous tau aggregation, which would prevent further modification of the protein by phosphorylation [22]. Interestingly, intracellular T3RC fragments are phosphorylated in PHF-1 domain (Fig. 3b), while extracellular T3RC is not recognize by the antibody PHF- 1 . This suggest that T3RC can be dephosphorylated extracellularly or that phosphorylated tau is not secreted. Interestingly, and as has been described for full-length tau in mature cortical neurons [38], phosphorylation of extracellular tau appears to be reduced in comparison with intracellular tau. Also, it has been described that an extracellular phosphatase could act on phosphotau [13].

\section{Conclusion}

Thus, using a non-neuronal cell model, here we have attempted to unravel some of the mechanisms underlying constitutive and pathological tau secretion. This study shows that T2N and T3RC peptides are secreted to the extracellular medium although they do not share any region that could be involved in the release. It suggests different mechanisms for tau secretion. One of them would depend on $\mathrm{N}$-terminal region of tau molecule while in the absence of $\mathrm{N}$-terminal region, tau secretion could depend on its aggregation. These findings may have important implications to understand how tau is released outside a cell.

\section{Conflict of interest}

The authors have no financial, personal, or other conflicts of interest related to this work.

\section{Acknowledgements}

This study was funded by grants from the Ministerio de Economia y Competitividad (SAF2014-53040-P and BUF2013-40664-P), the Centro de Investigación en Red de Enfermedades Neurodegenerativas (CIBERNED, ISCIII, JA) and the Comunidad de Madrid (S2010/BMD-2331). We also acknowledge institutional support from the Fundación Ramón Areces. The authors thank Nuria de la Torre for her technical assistance.

\section{References}

[1] G. Amadoro, V. Corsetti, G.M. Sancesario, A. Lubrano, G. Melchiorri, S Bernardini, P. Calissano, G. Sancesario, Cerebrospinal fluid levels of a 20-22 kDa NH2 fragment of human tau provide a novel neuronal injury biomarker in Alzheimer's disease and other dementias, J. Alzheimers Dis. 42 (2014) 211-226.

[2] M. Arrasate, M. Perez, J. Avila, Tau dephosphorylation at tau-1 site correlates with its association to cell membrane, Neurochem. Res. 25 (2000) 43-50.

[3] J. Avila, J.J. Lucas, M. Perez, F. Hernandez, Role of tau protein in both physiological and pathological conditions, Physiol. Rev. 84 (2004) 361-384.

[4] N.R. Barthelemy, A. Gabelle, C. Hirtz, F. Fenaille, N. Sergeant, S. Schraen-Maschke, J. Vialaret, L. Buee, C. Junot, F. Becher, S. Lehmann, Differential mass spectrometry profiles of tau protein in the cerebrospinal fluid of patients with Alzheimer's disease, progressive supranuclear palsy, and dementia with lewy bodies, J. Alzheimers Dis. 51 (2016) 1033-1043.

[5] A.J. Barton, P.J. Harrison, A. Najlerahim, J. Heffernan, B. McDonald, J.R. Robinson, D.C. Davies, W.J. Harrison, P. Mitra, J.A. Hardy, et al., Increased tau messenger RNA in Alzheimer's disease hippocampus, Am. J. Pathol. 137 (1990) 497-502

[6] G. Basurto-Islas, J. Luna-Munoz, A.L. Guillozet-Bongaarts, L.I. Binder, R. Mena, F. Garcia-Sierra, Accumulation of aspartic acid421- and glutamic acid391-cleaved tau in neurofibrillary tangles correlates with progression in Alzheimer disease, J. Neuropathol. Exp. Neurol. 67 (2008) 470-483.

[7] K. Blennow, A. Wallin, H. Agren, C. Spenger, J. Siegfried, E. Vanmechelen, Tau protein in cerebrospinal fluid: a biochemical marker for axonal degeneration in Alzheimer disease? Mol. Chem. Neuropathol. 26 (1995) 231-245.

[8] R. Brandt, J. Leger, G. Lee, Interaction of tau with the neural plasma membrane mediated by tau's amino-terminal projection domain, J. Cell Biol. 131 (1995) 1327-1340.

[9] F. Clavaguera, T. Bolmont, R.A. Crowther, D. Abramowski, S. Frank, A. Probst G. Fraser, A.K. Stalder, M. Beibel, M. Staufenbiel, M. Jucker, M. Goedert, M. Tolnay, Transmission and spreading of tauopathy in transgenic mouse brain, Nat. Cell Biol. 11 (2009) 909-913.

[10] V. Corsetti, F. Florenzano, A. Atlante, A. Bobba, M.T. Ciotti, F. Natale, F. Della Valle, A. Borreca, A. Manca, G. Meli, C. Ferraina, M. Feligioni, S. D’Aguanno, R. Bussani, M. Ammassari-Teule, V. Nicolin, P. Calissano, G. Amadoro, NH2-truncated human tau induces deregulated mitophagy in neurons by aberrant recruitment of Parkin and UCHL-1: implications in Alzheimer's disease, Hum. Mol. Genet. 24 (2015) 3058-3081.

[11] X. Chai, J.L. Dage, M. Citron, Constitutive secretion of tau protein by an unconventional mechanism, Neurobiol. Dis. 48 (2012) 356-366.

[12] P. Delobel, I. Lavenir, G. Fraser, E. Ingram, M. Holzer, B. Ghetti, M.G. Spillantini, R.A. Crowther, M. Goedert, Analysis of tau phosphorylation and truncation in a mouse model of human tauopathy, Am. J. Pathol. 172 (2008) 123-131.

[13] M. Diaz-Hernandez, A. Gomez-Ramos, A. Rubio, R. Gomez-Villafuertes, J.R. Naranjo, M.T. Miras-Portugal, J. Avila, Tissue-nonspecific alkaline phosphatase promotes the neurotoxicity effect of extracellular tau, J. Biol. Chem. 285 (2010) 32539-32548.

[14] S. Dujardin, S. Begard, R. Caillierez, C. Lachaud, L. Delattre, S. Carrier, A. Loyens, M.C. Galas, L. Bousset, R. Melki, G. Auregan, P. Hantraye, E. Brouillet, L. Buee, M. Colin, Ectosomes: a new mechanism for non-exosomal secretion of tau protein, PLoS One 9 (2014) e100760.

[15] S. Dujardin, K. Lecolle, R. Caillierez, S. Begard, N. Zommer, C. Lachaud, S. Carrier, N. Dufour, G. Auregan, J. Winderickx, P. Hantraye, N. Deglon, M. Colin, L. Buee, Neuron-to-neuron wild-type Tau protein transfer through a trans-synaptic mechanism: relevance to sporadic tauopathies, Acta Neuropathol. Commun. 2 (2014) 14.

[16] B. Frost, R.L. Jacks, M.I. Diamond, Propagation of tau misfolding from the outside to the inside of a cell, J. Biol. Chem. 284 (2009) 12845-12852.

[17] Y. Gluzman, SV40-transformed simian cells support the replication of early SV40 mutants, Cell 23 (1981) 175-182.

[18] M. Goedert, NEURODEGENERATION. Alzheimer's and Parkinson's diseases: the prion concept in relation to assembled Abeta, tau, and alpha-synuclein, Science 349 (2015) 1255555 
[19] M. Goedert, M.G. Spillantini, M.C. Potier, J. Ulrich, R.A. Crowther, Cloning and sequencing of the cDNA encoding an isoform of microtubule-associated protein tau containing four tandem repeats: differential expression of tau protein mRNAs in human brain, EMBO J. 8 (1989) 393-399.

[20] A. Gomez-Ramos, M. Diaz-Hernandez, R. Cuadros, F. Hernandez, J. Avila, Extracellular tau is toxic to neuronal cells, FEBS Lett. 580 (2006) 4842-4850.

[21] G.F. Hall, S. Saman, Death or secretion? The demise of a plausible assumption about CSF-tau in Alzheimer Disease? Commun. Integr. Biol. 5 (2012) 623-626.

[22] D.P. Hanger, J.C. Betts, T.L. Loviny, W.P. Blackstock, B.H. Anderton, New phosphorylation sites identified in hyperphosphorylated tau (paired helical filament-tau) from Alzheimer's disease brain using nanoelectrospray mass spectrometry, J. Neurochem. 71 (1998) 2465-2476.

[23] M. Hasegawa, Molecular mechanisms in the pathogenesis of Alzheimer's disease and tauopathies-prion-like seeded aggregation and phosphorylation, Biomolecules 6 (2016).

[24] S.C. Hwang, D.Y. Jhon, Y.S. Bae, J.H. Kim, S.G. Rhee, Activation of phospholipase C-gamma by the concerted action of tau proteins and arachidonic acid, J. Biol. Chem. 271 (1996) 18342-18349.

[25] K. Iqbal, F. Liu, C.X. Gong, I. Grundke-Iqbal, Tau in Alzheimer disease and related tauopathies, Curr. Alzheimer Res. 7 (2010) 656-664.

[26] D. Kanmert, A. Cantlon, C.R. Muratore, M. Jin, T.T. O’Malley, G. Lee, T.L. Young-Pearse, D.J. Selkoe, D.M. Walsh, C-terminally truncated forms of tau, but not full-length tau or its C-terminal fragments are released from neurons independently of cell death, J. Neurosci. 35 (2015) 10851-10865.

[27] W. Kim, S. Lee, G.F. Hall, Secretion of human tau fragments resembling CSF-tau in Alzheimer's disease is modulated by the presence of the exon 2 insert, FEBS Lett. 584 (2010) 3085-3088.

[28] M.N. Le, W. Kim, S. Lee, A.C. McKee, G.F. Hall, Multiple mechanisms of extracellular tau spreading in a non-transgenic tauopathy model, Am. J. Neurodegener. Dis. 1 (2012) 316-333.

[29] V. Malhotra, Unconventional protein secretion: an evolving mechanism, EMBO J. 32 (2013) 1660-1664.

[30] S.E. Matsumoto, Y. Motoi, K. Ishiguro, T. Tabira, F. Kametani, M. Hasegawa, N. Hattori, The twenty-four kDa C-terminal tau fragment increases with aging in tauopathy mice: implications of prion-like properties, Hum. Mol. Genet. 24 (2015) 6403-6416.

[31] M. Medina, E. Montejo de Garcini, J. Avila, The role of tau phosphorylation in transfected COS-1 cells, Mol. Cell. Biochem. 148 (1995) 79-88.

[32] N.V. Mohamed, T. Herrou, V. Plouffe, N. Piperno, N. Leclerc, Spreading of tau pathology in Alzheimer's disease by cell-to-cell transmission, Eur. J. Neurosci. 37 (2013) 1939-1948

[33] N.V. Mohamed, V. Plouffe, G. Remillard-Labrosse, E. Planel, N. Leclerc, Starvation and inhibition of lysosomal function increased tau secretion by primary cortical neurons, Sci. Rep. 4 (2014) 5715.

[34] E. Montejo de Garcini, S. de la Luna, J.E. Dominguez, J. Avila, Overexpression of tau protein in COS-1 cells results in the stabilization of centrosome-independent microtubules and extension of cytoplasmic processes, Mol. Cell. Biochem. 130 (1994) 187-196.

[35] S. Ozcelik, F. Sprenger, Z. Skachokova, G. Fraser, D. Abramowski, F. Clavaguera, A. Probst, S. Frank, M. Muller, M. Staufenbiel, M. Goedert, M. Tolnay, D.T. Winkler, Co-expression of truncated and full-length tau induces severe neurotoxicity, Mol. Psychiatry (2016), http://dx.doi.org/10.1038/mp.2015. 228.

[36] V. Plouffe, N.V. Mohamed, J. Rivest-McGraw, J. Bertrand, M. Lauzon, N. Leclerc, Hyperphosphorylation and cleavage at D421 enhance tau secretion, PLoS One 7 (2012) e36873.

[37] J.C. Polanco, B.J. Scicluna, A.F. Hill, J. Gotz, Extracellular vesicles isolated from brains of rTg4510 mice seed tau aggregation in a threshold-dependent manner, J. Biol. Chem. 291 (2016) 12445-12466.

[38] A.M. Pooler, E.C. Phillips, D.H. Lau, W. Noble, D.P. Hanger, Physiological release of endogenous tau is stimulated by neuronal activity, EMBO Rep. 14 (2013) 389-394

[39] M. Riemenschneider, S. Wagenpfeil, H. Vanderstichele, M. Otto, J. Wiltfang, H. Kretzschmar, E. Vanmechelen, H. Forstl, A. Kurz, Phospho-tau/total tau ratio in cerebrospinal fluid discriminates Creutzfeldt-Jakob disease from other dementias, Mol. Psychiatry 8 (2003) 343-347.

[40] S. Saman, W. Kim, M. Raya, Y. Visnick, S. Miro, S. Saman, B. Jackson, A.C. McKee, V.E. Alvarez, N.C. Lee, G.F. Hall, Exosome-associated tau is secreted in tauopathy models and is selectively phosphorylated in cerebrospinal fluid in early Alzheimer disease, J. Biol. Chem. 287 (2012) 3842-3849.

[41] D. Simon, E. Garcia-Garcia, F. Royo, J.M. Falcon-Perez, J. Avila, Proteostasis of tau. Tau overexpression results in its secretion via membrane vesicles, FEBS Lett. 586 (2012) 47-54

[42] S. Sokolow, K.M. Henkins, T. Bilousova, B. Gonzalez, H.V. Vinters, C.A. Miller, L. Cornwell, W.W. Poon, K.H. Gylys, Pre-synaptic C-terminal truncated tau is released from cortical synapses in Alzheimer's disease, J. Neurochem. 133 (2015) 368-379.

[43] M.D. Weingarten, A.H. Lockwood, S.Y. Hwo, M.W. Kirschner, A protein factor essential for microtubule assembly, Proc. Natl. Acad. Sci. U. S. A. 72 (1975) 1858-1862.

[44] K. Yamada, J.R. Cirrito, F.R. Stewart, H. Jiang, M.B. Finn, B.B. Holmes, L.I. Binder E.M. Mandelkow, M.I. Diamond, V.M. Lee, D.M. Holtzman, In vivo microdialysis reveals age-dependent decrease of brain interstitial fluid tau levels in P301S human tau transgenic mice, J. Neurosci. 31 (2011) $13110-13117$.

[45] Z. Zhang, M. Song, X. Liu, S.S. Kang, I.S. Kwon, D.M. Duong, N.T. Seyfried, W.T Hu, Z. Liu, J.Z. Wang, L. Cheng, Y.E. Sun, S.P. Yu, A.I. Levey, K. Ye, Cleavage of tau by asparagine endopeptidase mediates the neurofibrillary pathology in Alzheimer's disease, Nat. Med. 20 (2014) 1254-1262. 